\title{
Large sphenoorbital meningioma with extension into the cavernous sinus
}

\author{
Nitin K Sethi ${ }^{*}$, Tomoko Ikuine, Nicolle Steiner \\ Department of Neurology, New York-Presbyterian Hospital, Weill Cornell Medical Center, New York, NY (U.S.A.)
}

Key Words: Parasellar meningioma, cavernous sinus

A-71-year-old right handed male presented for evaluation of episodes of fainting. He gave history of progressive visual loss in the left eye, diplopia and proptosis first noticed in the 1990's when a scan had revealed a left cavernous sinus tumor. He was lost to follow up subsequently gradually ignoring his visual deficits and continued to drive. Magnetic resonance imaging (MRI) of the brain now showed a large left parasellar mass, involving the left cavernous sinus and extending into the sella, with distortion of the canalicular, cisternal, and proximal orbital segment of the optic nerve and proptosis. Extension into the left middle fossa with mass effect on the medial left temporal lobe and pituitary tissue was present. The lesion near circumferentially involved the left cavernous carotid artery without significant narrowing.

Signal characteristics favored a slowly progressive sphenoorbital meningioma (Figure 1, 2 and 3). It was felt that the only clear indication for surgery now would be evidence of involvement of the right eye and so it was decided to follow him with periodic detailed neuro-ophthalmological evaluations (1).
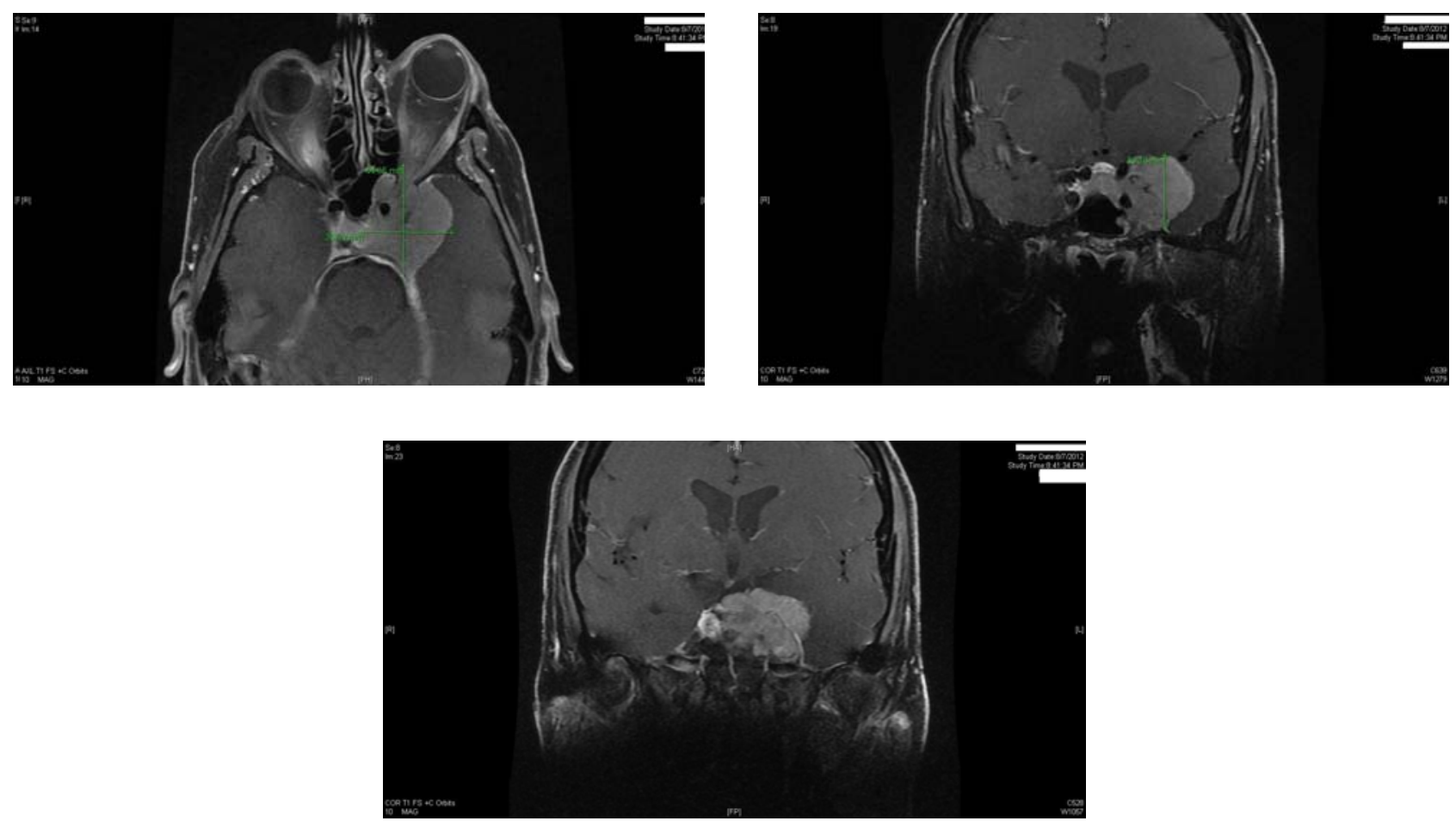

Fig. 1,2,3. Brain and orbits MRI images axial and coronal T1 FS with contrast show a large left sphenoorbital meningioma, involving the left cavernous sinus and extending into the sella, with distortion of the canalicular, cisternal, and proximal orbital segment of the optic nerve and proptosis. Extension into the left middle fossa with mass effect on the medial left temporal lobe and pituitary tissue is noted. The lesion near circumferentially involves the left cavernous carotid artery without significant narrowing.

${ }^{*}$ Corresponding Author: Nitin K. Sethi, MD. Comprehensive Epilepsy Center, New York-Presbyterian Hospital, Weill Cornell Medical Center 525 East, 68th Street New York, NY 10065, NY (U.S.A.) Tel: + 212-746-23 46, Fax: + 212-746-88 45 
Sethi et al / Meningioma of the cavernous sinus

\section{Reference}

1. Knosp E, Perneczky A, Koos WT, Fries G, Matula C. Meningiomas of the space of the cavernous sinus. Neurosurgery 1996; 38 : 434-442.

East J Med Volume:21, Number:4, October-December/2016 